\title{
GEM Detector Performance Assessment in the BM@N Experiment
}

\author{
Mikhail Kapishin ${ }^{1, \star}$, Vladimir Karjavin ${ }^{1}$, Elena Kulish ${ }^{1}$, Vasilisa Lenivenko ${ }^{1, \star \star}$, \\ Alexander Makankin ${ }^{1}$, Anna Maksymchuk ${ }^{1}$, Vladimir Palichik ${ }^{1}$, and Sergey Vasiliev ${ }^{1}$ \\ ${ }^{1}$ Joint Institute for Nuclear Research, Joliot Curie 6, 141980 Dubna, Moscow region, Russia
}

\begin{abstract}
The Gas Electron Multiplier (GEM) chambers are developed for modern purposes in the elementary particle physics. In the BM@N experiment, a GEM system is used for the reconstruction of the trajectories of the charged particles. The investigation of GEM performance (efficiency and spatial resolution) is presented.
\end{abstract}

\section{Introduction}

\subsection{Motivation}

The relativistic heavy ion collisions provide a unique opportunity to study the nuclear matter under extreme density and temperature. If the energy density in the formed fireball is sufficiently large the quark-gluon substructure of nucleons becomes visible. The existing theoretical models suggest different possible scenarios to describe the features of the strongly interacting matter. Experimental data at high detector resolution are needed in order to disentangle among the different theoretical predictions $[1,2]$ and to get the proper description of these phenomena.

\subsection{BM@N experiment}

The fixed target experiment BM@N (Baryonic Matter at Nuclotron) is the first step of the realization of the NICA project [3]. The main goals of the experiment are [4] the investigation of the nuclear equation-of-state, the study of the in-medium properties of the hadrons, the production of the multistrange hyperons at the threshold and the search for hypernuclei.

The experiment combines high precision track measurements with time-of-flight information for particle identification. The magnetic field of the analyzing magnet can be varied up to $1 \mathrm{~T}$ to get the optimal detector acceptance and momentum resolution for different reactions and beam energies. The charged particle momentum and multiplicity are measured with the set of the Gas Electron Multipliers (GEM) [5] located inside the analyzing magnet and by the Drift Chambers (DCH) and the Cathode Strip Chambers (CSC) situated outside the magnetic field.

${ }^{\star}$ e-mail: kapishin@jinr.ru

$\star \star$ e-mail: vasilisa@jinr.ru 


\subsection{Structure of the Gaseous Electron Multiplier}

Detectors based on the GEM technology have been identified as appropriate for the BM@N tracking system for their capability of stable operation in conditions of high beam intensities up to $10^{6}$ ions per second and in a strong magnetic field up to 1 T. The BM@N GEM chambers consist of three GEM multipliers, with the following gaps between the electrodes: the drift gap of $3 \mathrm{~mm}$, the first transfer gap of $2.5 \mathrm{~mm}$, the second transfer gap of $2 \mathrm{~mm}$ and the induction gap of $1.5 \mathrm{~mm}$.

Presently six GEM chambers are installed into the magnet: four "middle" chambers with the size of $66 \times 41 \mathrm{~cm}^{2}$ and two "large" chambers with the size of $163 \times 45 \mathrm{~cm}^{2}$. Each chamber has twocoordinate readout: along horizontal $X$-axis and along $X^{\prime}$ at angles \pm 15 degrees to the $X$-axis. The pitch of readout strips is 800 microns.

\section{Track-segment building algorithm (magnetic field off) and testing with Monte-Carlo data}

Two layers (X-plane and X'-plane) compose chamber. Each chamber layer consists of anode strips. In each fired strip charge from electron avalanches is collected. A group of sequence strips is united into one cluster. The center of gravity of the cluster provides the coordinate of the reconstructed hit.

$$
x_{c}=\frac{\sum_{i=1}^{n} x_{i} A_{i}}{\sum_{i=1}^{n} x_{i}},
$$

where $n$ is the cluster width, $x_{i}$ and $A_{i}$ are the coordinate and amplitude of the signal of the strip with signal respectively, $x_{c}$ is the center of gravity. A charged particle track-segment is built collecting the $X$ and $X^{\prime}$ hits which are located along the straight line. In algorithm GEM hit efficiency per layer is calculated for events where track-segments were reconstructed and defined as the ratio:

$$
\frac{\text { Number of the reconstructed hits in all layers }}{\text { Number of layers }} \text {. }
$$

For testing the efficiency of calculation algorithm simple generator was used with established efficiency per layer $90 \%$. Obtained averaged efficiencies are in a good agreement with the established number. Generator with background $=5$ hits per layer and dependence of efficiency vs background are shown in the figure 1.

\section{Nuclotron data}

\subsection{GEM hit efficiency per layer}

The GEM hit efficiency per layer with Nuclotron data (C beam without target, $4.5 \mathrm{GeV} / \mathrm{n}$ ) found for different gas mixtures is shown on the figure 2. The efficiency is high (92-95\%), the difference between the two investigated gas mixtures is $2-3 \%$.

For the two gas mixtures the cluster (a group of strips with signal) width is different. From Ar + $\mathrm{CO}_{2}$ cluster width is 2.3 strips in average. Most of clusters have only one strip. Cluster width for two gas mixtures is presented in figure 3 . 

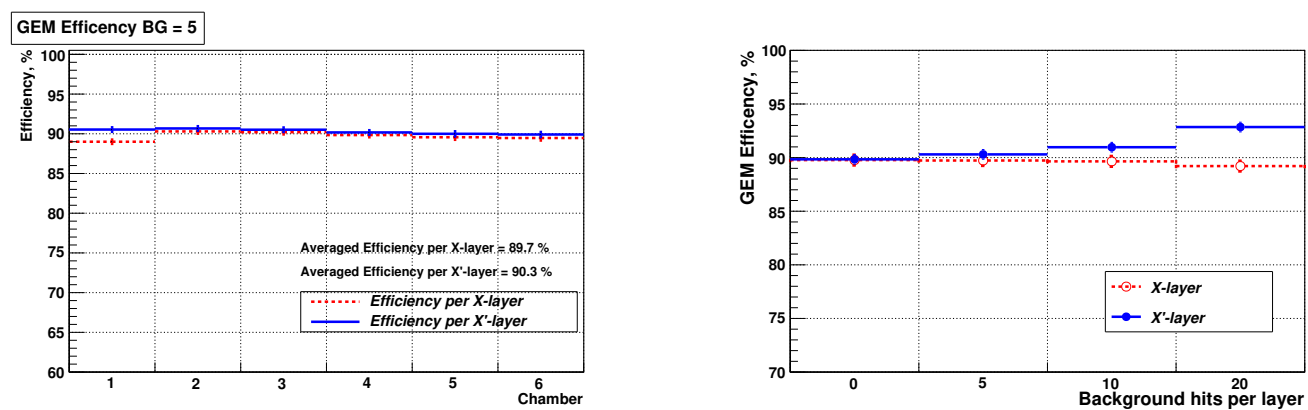

Figure 1. Resolved efficiency per chamber (left), dependence of efficiency vs background hits per layer (right) for simulated data. Simulated efficiency is $90 \%$ per layer.
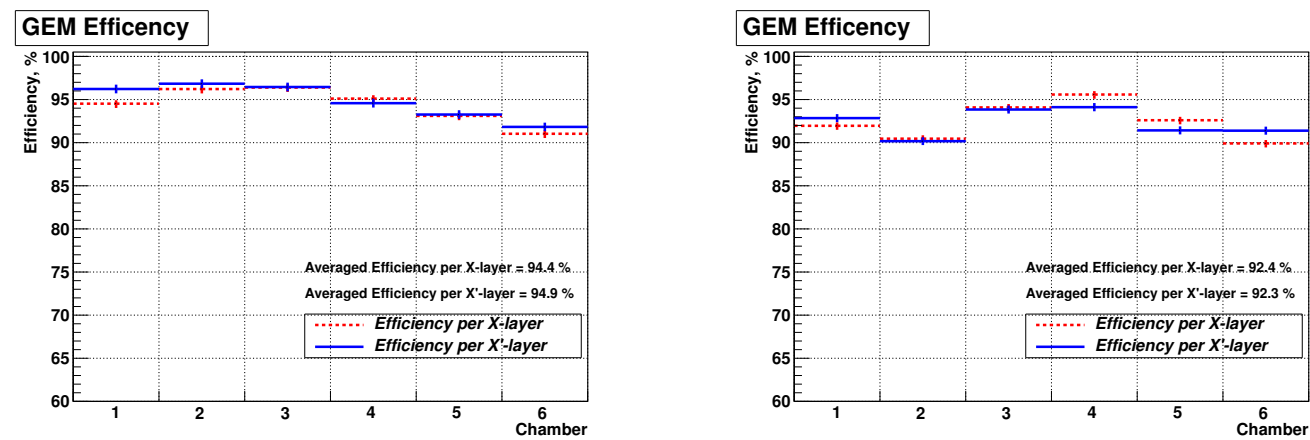

Figure 2. Efficiency per layer for two gas mixtures: $\mathrm{Ar}+\mathrm{CO}_{2}$ (left) and $\mathrm{Ar}+\mathrm{Isobutane}$ (right).
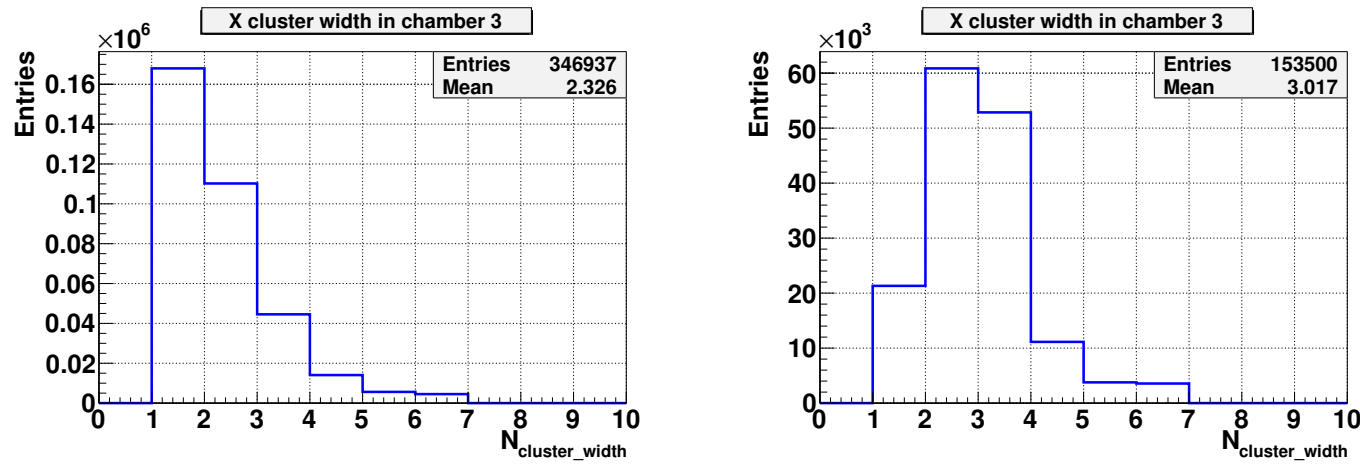

Figure 3. Cluster width in chamber 3 for two gas mixtures: $\mathrm{Ar}+\mathrm{CO}_{2}$ (left) and $\mathrm{Ar}+\mathrm{Isobutane}$ (right).

\subsection{GEM spatial resolution}

The spatial resolution was calculated from the residuals between the measurements and the fitted predictions in each layers. Results obtained for the $\mathrm{Ar}+\mathrm{CO}_{2}$ gas mixture are presented in the figure 4 . Obtained resolution about $200 \mu \mathrm{m}$ is similar to the one-strip clusters resolution, that should be $d / \sqrt{12}=$ $231 \mu \mathrm{m}$ with strip pitch equal $800 \mu \mathrm{m}$. This leads to the drop in presicion of the $X$ and $X^{\prime}$ coordinates reconstruction. Figure 5 illustrates the spatial resolution for the $\mathrm{Ar}+$ Isobutane gas mixture. The obtained resolution of $90-120 \mu \mathrm{m}$ is much better than that for $\mathrm{Ar}+\mathrm{CO}_{2}$ gas mixture, because the 
cluster width for the Ar + Isobutane gas mixture is in average 3 strips (mainly two- and three-strips clusters).
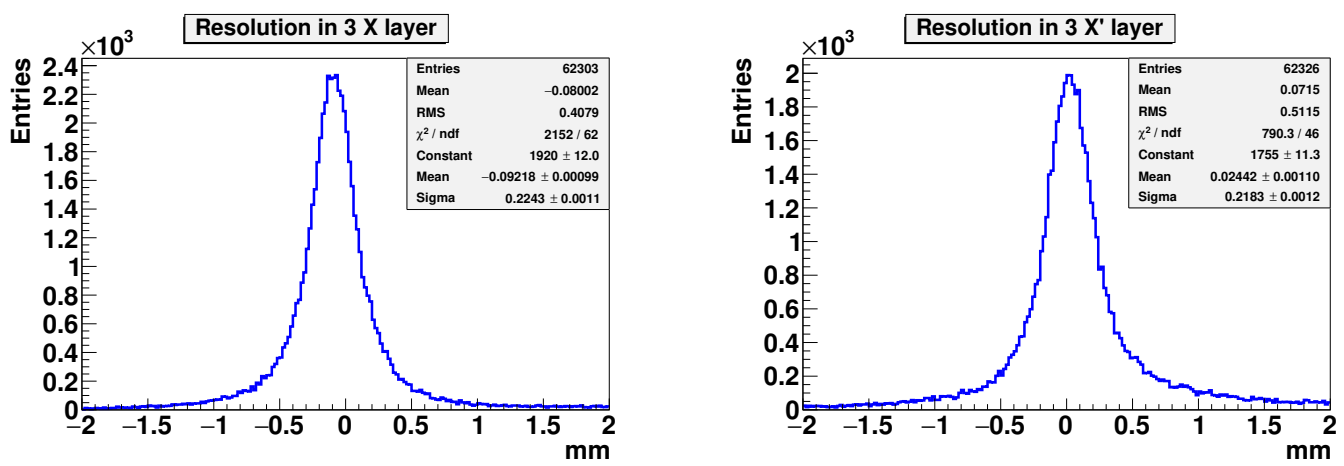

Figure 4. GEM chamber $3 X$ and $X^{\prime}$ resolution for $\mathrm{Ar}+\mathrm{CO}_{2}$ gas mixture. RMS are $0.3306 \mathrm{~mm}$ and $0.3113 \mathrm{~mm}$ accordingly.
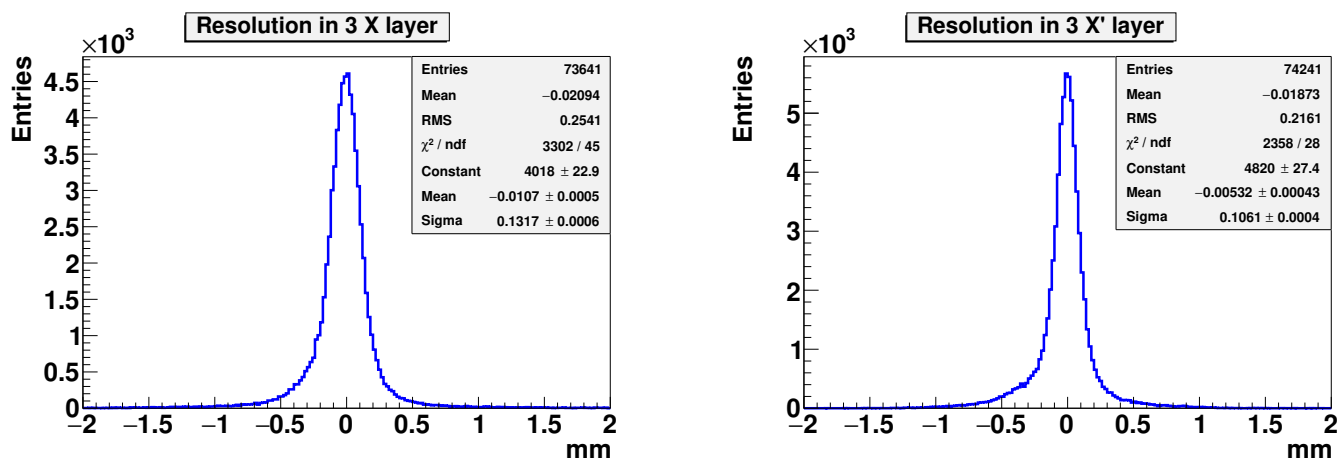

Figure 5. GEM chamber $3 X$ and $X^{\prime}$ resolution for $\mathrm{Ar}+$ Isobutane gas mixture. RMS are $0.2541 \mathrm{~mm}$ and 0.2161 mm accordingly.

\section{Conclusions}

The GEM chambers performance in the BM@N experiment was assessed for the Nuclotron data $(\mathrm{C}$ beam without target, $4.5 \mathrm{GeV} / \mathrm{n}$ ): satisfactory hit efficiency per layer $92-95 \%$, a spatial resolution of around $200 \mu \mathrm{m}$ for $\mathrm{Ar}+\mathrm{CO}_{2}$ gas mixture and a good spatial resolution of 90-120 $\mu \mathrm{m}$ for $\mathrm{Ar}+$ Isobutane gas mixture. Due to better spatial resolution at almost the same efficiency $\mathrm{Ar}+$ Isobutan gas mixture will be chosen to fill GEM chambers.

\section{References}

[1] J. Adams et al, Nucl. Phys. A 757, 102-183 (2005)

[2] K. Adcox et al., Nucl. Phys. A 757, 184-283 (2005)

[3] A.N. Sissakian et al. (NICA Collaboration), J. Phys. G36 064069 (2009)

[4] M. Kapishin, Eur. Phys. J. A 52, 213-219 (2016)

[5] F. Sauli, Nucl. Instrum. Meth. A 805, 2-24 (2016) 